\title{
Imager Device
}

National Cancer Institute

\section{Source}

National Cancer Institute. Imager Device. NCI Thesaurus. Code C49997.

A device designed to create or record a visual representation of something. 